\title{
Donor-specific anti-HLA antibodies are not associated with nonanastomotic biliary strictures but both are independent risk factors for graft loss after liver transplantation
}

\author{
Anne Claire den Dulk ${ }^{1}$ | Xiaolei Shi ${ }^{2}$ | Cornelia. J. Verhoeven ${ }^{3}$ | Jeroen Dubbeld ${ }^{4}$ | \\ Frans H. J. Claas $^{5}$ | Ron Wolterbeek ${ }^{6}$ | Simone H. Brand-Schaaf ${ }^{5}$ | \\ Hein W. Verspaget ${ }^{1}$ | Arantza Fariña Sarasqueta ${ }^{7}$ | Luc J. W. van der Laan ${ }^{3}$ | \\ Herold J. Metselaar $^{2}$ | Bart van Hoek ${ }^{1}$ (D) | Jaap Kwekkeboom ${ }^{2}$ | Dave L. Roelen ${ }^{5}$
}

\author{
${ }^{1}$ Department of Gastroenterology and \\ Hepatology, Leiden University Medical Center, \\ Leiden, The Netherlands \\ ${ }^{2}$ Department of Gastroenterology and \\ Hepatology, Erasmus MC-University Medical \\ Center, Rotterdam, The Netherlands \\ ${ }^{3}$ Department of Surgery, Erasmus \\ MC-University Medical Center, Rotterdam, \\ The Netherlands \\ ${ }^{4}$ Department of Transplant Surgery, Leiden \\ University Medical Center, Leiden, \\ The Netherlands \\ ${ }^{5}$ Department of Immunohematology and \\ Blood Transfusion, Section Immunogenetics \\ and Transplantation Immunology, Leiden \\ University Medical Center, Leiden, \\ The Netherlands \\ ${ }^{6}$ Department of Medical Statistics and \\ Bioinformatics, Leiden University Medical \\ Center, Leiden, The Netherlands \\ ${ }^{7}$ Department of Pathology, Leiden University \\ Medical Center, Leiden, The Netherlands \\ Correspondence \\ Bart van Hoek, MD, PhD, Department of \\ Gastroenterology and Hepatology, Leiden \\ University Medical Center, Leiden, \\ The Netherlands. \\ Email: B.van_Hoek@lumc.nl
}

Funding information

Anne Claire den Dulk was supported by a grant from Fund NutsOhra and Xiaolei Shi was supported by a PhD fellowship grant of the China Scholarship Council (File No. 2011623039)

\begin{abstract}
Donor-specific alloantibodies (DSA) have been associated with rejection and shorter graft survival after orthotopic liver transplantation (OLT). We examined the role of DSA in nonanastomotic biliary strictures (NAS) after OLT. Patients receiving first OLT who developed NAS $(n=68)$ and a control group without NAS $(n=83)$, with pre-OLT and 12 months post-OLT serum samples, were included. DSA were specified using the Luminex single antigen test. Risk factors for NAS and graft survival were analyzed. The presence of preformed DSA was not significantly different between patients with NAS and controls $(P=.89)$. After 12 months, $26.5 \%$ of NAS patients and $16.9 \%$ of controls had generated de novo DSA $(P=.15)$. Neither de novo class I DSA nor de novo class II DSA were associated with NAS. De novo DSA generally developed after the diagnosis of NAS. Time-dependent regression analysis identified both NAS (aHR 8.05, Cl 3.28 - 19.77, $P<.01$ ) and de novo class II DSA (aHR 2.84, CI $1.38-5.82, P<.01$ ) as independent risk factors for graft loss. Preformed or de novo DSA were not associated with the development of NAS. However, NAS as well as de novo class II DSA were independent risk factors for graft loss after OLT.
\end{abstract}

\section{KEYWORDS}

biliary strictures, complications, donor-specific antibodies, HLA-antibody post-transplantation, liver transplantation, outcome

den Dulk and Shi share first authorship.

This is an open access article under the terms of the Creative Commons Attribution-NonCommercial License, which permits use, distribution and reproduction in any medium, provided the original work is properly cited and is not used for commercial purposes.

(c) 2017 The Authors. Clinical Transplantation Published by John Wiley \& Sons Ltd 


\section{1 | INTRODUCTION}

The presence of donor-specific alloantibodies (DSA) against human leukocyte antigen (HLA) has been associated with (hyper)acute rejection and shorter graft survival after organ transplantation in general. ${ }^{1,2}$ Yet, after orthotopic liver transplantation (OLT), preformed DSA can be absorbed by the graft and hyperacute rejection as a result of DSA is rare. ${ }^{3-5}$ While $A B O$ compatibility is required, the presence of DSA or a positive HLA crossmatch is not considered a contraindication to OLT. $^{6}$ Despite the absence of hyperacute rejection, recent studies have shown that DSA may be a more relevant predictor of patient and graft outcome after OLT than previously assumed. In a large study cohort, preformed class II DSA were associated with both early graft loss and rejection. ${ }^{7}$ In addition, Kaneku et al recently found that de novo DSA, formed after OLT, were detected in $8 \%$ of the patients and were associated with a significantly impaired patient and graft survival. ${ }^{8}$ Furthermore, it has been suggested that de novo DSA with a high mean fluorescence intensity (MFI) are associated with chronic rejection after OLT. ${ }^{9}$

Nonanastomotic biliary strictures (NAS) can be defined as intraand extrahepatic lesions of the biliary tree more than $1 \mathrm{~cm}$ above the anastomosis, characterized by bile duct strictures and dilatations. ${ }^{10,11}$ Reported incidences of treated NAS vary between $9 \%$ and $31 \%$. NAS are considered a major cause of morbidity and reduced graft survival after OLT. $^{12}$ Whereas the complete pathogenesis of NAS is still unclear, several risk factors such as ischemia-reperfusion injury, ${ }^{13}$ grafts donated after cardiac death and damage from bile salt toxicity have been identified. ${ }^{14,15}$ Besides these factors, a contribution of immune-mediated cholangiocyte injury has been proposed. ${ }^{16}$ This is supported by reports on an increased incidence of NAS in the cases of $A B O$ incompatibility, underlying disease with assumed autoimmune etiology-such as primary sclerosing cholangitis (PSC) and autoimmune hepatitis-in patients with a genetic chemokine receptor five loss of function, and after cytomegalovirus viremia (CMV). ${ }^{17-20}$ The biliary epithelium has been shown to express HLA class I and class II molecules after $\mathrm{OLT}^{21}$ and the presence of antibodies against these antigens may therefore possibly be a factor contributing to NAS development. Indeed, earlier studies demonstrated that the presence of preformed DSA leading to a positive cytotoxic crossmatch was associated with bile duct complications in general and/or with preservation injury, which is an important risk factor for NAS development. ${ }^{10,22-25}$ Therefore, the aim of this study was to evaluate the relationship between preformed and de novo DSA with the development of NAS and with graft survival after OLT.

\section{2 | PATIENTS AND METHODS}

Patients receiving ABO-compatible OLT in two Dutch transplantation centers between 2000 and 2014 who developed NAS $(n=68)$ and a control group of OLT patients without NAS matched for recipient age, recipient gender, etiology, and acute rejection, and transplanted in the same time period $(n=83)$, of whom pre-OLT and 12 months post-OLT serum samples were available, were selected for this study. Duration of follow-up was at least 1 year for all patients. Presentation was with bacterial cholangitis, jaundice and/or itching in around one-third of cases, all with cholestatic liver enzymes. In two-thirds of patients, no symptoms were present, while elevated serum alkaline phosphatase and gammaglutamyltransferase, and bilirubin in some, prompted further investigation. In all NAS patients, the diagnosis was confirmed with direct cholangiography, that is, endoscopic retrograde cholangiography or percutaneous transhepatic cholangiography. In all cases diagnosed with NAS, biliary strictures more than $1 \mathrm{~cm}$ above the biliary anastomosis were present as determined by at least two endoscopists and conformed in a multidisciplinary radiology meeting. Moreover, a strict definition of NAS was used, which required that the biliary strictures should have been treated at least once by dilatation and/or stenting by ERCP or PTC, ensuring that the strictures were considered clinically significant. In this cohort, only a minority underwent MRI-MRCP first. In all of these cases, NAS was intrahepatic and most often diffuse. Some were only perihilar, a minority in addition had extrahepatic nonanastomotic biliary strictures. Only patients without vascular (eg, hepatic artery thrombosis) or other biliary complications were included. Hepatic artery thrombosis or stenosis and other complications were excluded by Doppler ultrasound or CT angiography in all cases.

Graft survival was determined as time of transplantation until graft loss (retransplantation or death) or in case of no event, until the end of the study (June 2014). Time to NAS was determined as time from transplantation until detection of NAS or in case of no event, until graft loss or the end of the study (June 2014). Demographic and clinical data of liver transplant recipients, like age, gender, etiology of liver disease and post-transplant complications, were derived from the electronic patient charts. Immunosuppression in both centers was similar, consisting of induction with basiliximab and methylprednisolone, followed by tacrolimus, or-in some cases-cyclosporin microemulsion, prednisolone for 6 months and in some cases addition of mycophenolate mofetil, azathioprine or-after 3 months-everolimus or sirolimus.

The study was approved by the Medical Ethics Committee (Protocol B14.014) and in accordance with the Declaration of Helsinki. All patients gave informed consent to donate pretransplantation and post-transplantation blood samples for research purposes, without given preference to any explicit clinical variables. Only patients with a minimum age of 18 years who gave informed consent and donated a blood sample were included in the study.

\section{1 | Human leukocyte antigen typing and determination of anti-HLA class I and class II alloantibodies}

Patient and donor DNA samples were genotyped using either the sequence-specific oligonucleotide probe (PCR/SSOP) technique for HLA-DR and HLA-DQ, or the reverse SSO method on a suspension array platform using microspheres as a solid support to 
immobilize oligonucleotide probes (for HLA-A and HLA-B: Lifecodes from Immucor Transplant Diagnostics Inc., Stamford, CT, USA). Serum samples from recipients were screened for the presence of anti-HLA alloantibodies using the Lifecodes Lifescreen Deluxe (LMX) kit, according to the manufacturer's manual (Immucor Transplant Diagnostics Inc.). Samples that were positive for either HLA class I (HLA-A or HLA-B) or HLA class II (HLA-DQ or HLA-DR) antibodies were further analyzed with a Luminex single antigen assay, using LABscreen HLA class I and class II antigen beads (One Lambda, Canoga Park, CA, USA). Briefly, $4 \mu \mathrm{L}$ of LABscreen beads and $20 \mu \mathrm{L}$ of serum were mixed in a test well, protected from light. Serum samples were incubated for 30 minutes. at room temperature on a rotating platform (150 rpm), followed by repeated washings with $260 \mu \mathrm{L}$ wash buffer (1X). Afterward, each sample was incubated for 30 minutes. with a goat anti-human PE-conjugated antibody (1:100 wash buffer) at room temperature, protected from light, and subsequently washed five times with wash buffer. Samples were measured using a Luminex 100 reader (Luminex 100, Luminex Corporation, 's-Hertogenbosch, the Netherlands). LABscreen negative control serum (LS-NC, One Lambda) was used as a negative control. Antibodies detected with a MFI of $>5000$ were considered positive, as DSA levels above this cutoff value are associated with clinically relevant outcomes after OLT.,

\section{2 | Statistical analysis}

Statistical analysis was performed using SPSS version 20.0 for windows (SPSS Inc., Chicago, IL, USA). Continuous data were analyzed using Student $t$ test or Mann-Whitney $U$ test. Chi-square test was performed for categorized data. A risk factor analysis for NAS and for graft loss was performed using univariate and (for univariate factors with $P<.10)$ multivariate Cox regression analyses for baseline factors with forward selection and backward exclusion. Both NAS and de novo class II DSA were considered risk factors for graft loss, and as these predictors occur in the course of time, instead of being baseline variables, these factors were taken into account as time-dependent covariates in a time-dependent Cox regression analysis. A P-value of $<.05$ was considered statistically significant.

\section{3 | RESULTS}

\section{1 | Patients}

In total, 68 patients with nonanastomotic biliary strictures (NAS) and 83 controls without NAS after first OLT were included in this study. Median age of all recipients at time of transplantation was 55 (IQR 46-61) years. In 31 patients (20.5\%), OLT was performed with a graft from donation after circulatory death (DCD). Median time from OLT to diagnosis of NAS was 5.5 months (IQR 1.6-12.8). Baseline characteristics of patients with and without NAS are expressed in Table 1. No significant differences were found between patients with and without NAS, including the incidence of acute cellular rejection, with the exception of DCD-OLTs: As expected, NAS developed more frequently in recipients who received a DCD graft
TABLE 1 Baseline characteristics. Data are presented as median (interquartile range) for continuous variables. Categorized data are presented as number (percentage)

\begin{tabular}{|c|c|c|c|}
\hline & $\begin{array}{l}\text { NAS } \\
(n=68)\end{array}$ & $\begin{array}{l}\text { Controls } \\
(n=83)\end{array}$ & $P$-value \\
\hline Donor age & $47(37-55)$ & $47(36-58)$ & .980 \\
\hline Donor gender & & & .364 \\
\hline Male & $37(54.4)$ & $39(47.0)$ & \\
\hline Female & $31(45.6)$ & 44 (53.0) & \\
\hline Recipient age & $55(45-62)$ & $54.0(47-60)$ & .928 \\
\hline Recipient gender & & & .429 \\
\hline Male & $46(67.6)$ & $51(61.4)$ & \\
\hline Female & $22(32.4)$ & $32(38.6)$ & \\
\hline Etiology & & & .854 \\
\hline Viral & 7 (10.3) & 11 (13.3) & \\
\hline ALD & 13 (19.1) & $13(15.7)$ & \\
\hline PSC & $13(19.1)$ & $17(20.5)$ & \\
\hline $\mathrm{HCC}$ & $14(20.6)$ & $21(25.3)$ & \\
\hline Other $^{a}$ & 21 (30.9) & $21(25.3)$ & \\
\hline $\begin{array}{l}\text { CMV mismatch } \\
(\mathrm{D}+/ \mathrm{R}-)\end{array}$ & $28(47.5)$ & $44(53.7)$ & .467 \\
\hline $\begin{array}{l}\text { Episode of acute } \\
\text { rejection }\end{array}$ & $14(20.6)$ & $12(14.5)$ & .321 \\
\hline $\begin{array}{c}\text { Donation after } \\
\text { cardiac death }\end{array}$ & 24 (35.3) & $7(8.4)$ & .000 \\
\hline Donor BMI & $24(23-27)$ & $24(22-26)$ & .573 \\
\hline Recipient BMI & $25(23-28)$ & $25(23-28)$ & .731 \\
\hline $\begin{array}{l}\text { DWIT (min) in } \\
\text { DCD }\end{array}$ & $20(16-29)$ & $17(13-20)$ & .645 \\
\hline CIT (min) & $438(382-558)$ & $452(369-546)$ & .444 \\
\hline RWIT (min) & $33(26-42)$ & $31(24-34)$ & .148 \\
\hline
\end{tabular}

NAS, nonanastomotic biliary strictures; ALD, alcoholic liver disease; PSC, primary sclerosing cholangitis; HCC, hepatocellular carcinoma; CMV, cytomegalovirus; BMI, body mass index; DWIT, donor warm ischemia time; CIT, cold ischemia time; RWIT, recipient warm ischemia time. Significant values are printed bold.

${ }^{a}$ Other indications include: auto-immune hepatitis, nonalcoholic steatohepatitis and polycystic liver disease.

as compared to a liver after donation after brain death (DBD) donation $(P<.01)$.

\subsection{Donor-specific antibodies and NAS}

Preformed DSA were detected in $10.3 \%(n=7)$ of the NAS patients and in $9.6 \%(n=8)$ of the controls (Table 2). The presence of preformed class I DSA or preformed class II DSA was not significantly different between patients with NAS and controls in chi-square test. Female patients were more likely to have generated DSA before transplantation (female $20.4 \%$ vs male $4.1 \%, P<.01$ ). DSA did not develop more frequently in patients transplanted with a DCD graft (DBD 22.5\% vs DCD 16.1\%, $P=.44$ ). 1 year after OLT, 32 patients (21\%) generated de novo DSA. In 29 of 32 patients (90.6\%) who 
TABLE 2 Prevalence of preformed and de novo DSA (mean fluorescence intensity $[\mathrm{MFI}]>5000$ ) in patients with nonanastomotic biliary strictures (NAS) and controls

\begin{tabular}{|cccc|} 
& NAS $(\mathbf{n}=68)$ & Controls $(\mathbf{n}=83)$ & $P$-value \\
\hline Preformed & & & \\
\hline Any DSA & $7(10.3)$ & $8(9.6)$ & .893 \\
\hline Class I & $4(5)$. & $7(8.4)$ & .548 \\
\hline Class II & $2(2.9)$ & $1(1.2)$ & .447 \\
\hline Class I and II & $1(1.5)$ & 0 & .268 \\
\hline De novo & & & \\
\hline Any DSA & $18(26.5)$ & $14(16.9)$ & .151 \\
\hline Class I & $3(4.4)$ & 0 & .053 \\
\hline Class II & $15(22.1)$ & $14(16.9)$ & .420 \\
\hline
\end{tabular}

Data are presented as $\mathrm{n}(\%)$.

generated de novo DSA, the newly developed antibodies were directed against the HLA class II antigens of the donor, in most of the cases against HLA-DQ (69\% DQ only, 14\% DR only and $17 \%$ both DQ and DR). Overall, the development of DSA post-transplantation was not related to NAS development, as $26.5 \%$ of NAS patients and $16.9 \%$ of the controls had de novo DSA 1 year after OLT $(P=.15)$. Neither de novo class I DSA nor de novo class II DSA were significantly more often present in patients with NAS, as compared to controls (Table 2). The incidence of alloantibodies directed at antigens of the individual loci, that is, HLA-A, HLA-B, HLA-DQ and HLA-DR, was also not different between the two groups (data not shown). We further analyzed the cumulative MFI of DSA between patients with NAS and controls; however, no significant difference was found (13601 vs $10925, P=.27$ ).

Univariate and multivariate analyses of pre-OLT risk factors for developing NAS are shown in Table 3. As expected a DCD donor was a risk factor for NAS in both univariate and multivariate analyses, while a donor with a positive CMV IgG status was a risk factor in univariate analysis with only a trend in multivariate analysis. Preformed DSA overall and class I and II apart were not risk factors for NAS, and likewise, the other examined factors were not a risk for developing NAS (Table 3). Univariate and multivariate analyses for baseline risk factors were also examined after exclusion of DCD OLT: In DBD OLT, a trend existed for RWIT as a risk factor for NAS in both univariate and multivariate analyses, and no significant risk factors for NAS were found here (Table 4). In the whole cohort and in DBD OLT, there was also no relationship between DSA against various HLA antigens and NAS with MFI of $>1000$ (instead of $>5000$ ) as cutoff for the presence of DSA (Tables 3 and 4).

Longitudinal analysis in a subgroup of NAS patients $(n=20)$ showed that preexisting DSA against class I HLA usually disappeared within the first 6 months after transplantation. De novo class I DSA tended to develop early after OLT, but were usually already cleared

\begin{tabular}{|c|c|c|c|c|}
\hline & \multicolumn{2}{|l|}{ Univariate analysis } & \multicolumn{2}{|c|}{ Multivariate analysis } \\
\hline & HR $(95 \% \mathrm{Cl})$ & $P$-value & HR $(95 \% \mathrm{Cl})$ & $P$-value \\
\hline Donor age & $1.00(0.98-1.02)$ & .835 & & \\
\hline $\begin{array}{l}\text { Donor gender male } \\
\text { CMV IgG donor positive }\end{array}$ & $\begin{array}{l}0.93(0.56-1.59) \\
0.54(0.32-0.92)\end{array}$ & $\begin{array}{l}.826 \\
.023\end{array}$ & $1.61(0.93-2.78)$ & .088 \\
\hline $\begin{array}{l}\text { CMV mismatch ( } D \text { pos/R neg) } \\
\text { CMV IgG recipient positive }\end{array}$ & $\begin{array}{l}0.73(0.35-1.53) \\
0.77(0.42-1.43)\end{array}$ & $\begin{array}{l}.409 \\
.403\end{array}$ & & \\
\hline $\begin{array}{l}\text { Recipient age } \\
\text { Recipient gender male }\end{array}$ & $\begin{array}{l}1.01(0.99-1.04) \\
1.21(0.73-1.01)\end{array}$ & $\begin{array}{l}.404 \\
.472\end{array}$ & & \\
\hline $\begin{array}{l}\text { Gender mismatch } \\
\text { BMI donor }\end{array}$ & $\begin{array}{l}0.89(0.53-1.51) \\
1.01(0.93-1.09)\end{array}$ & $\begin{array}{l}.669 \\
.854\end{array}$ & & \\
\hline $\begin{array}{l}\text { OLT indication grouped } \\
\text { OLT indication PSC }\end{array}$ & $\begin{array}{r}(0.66-1.24) \\
1.36(0.61-2.13)\end{array}$ & $\begin{array}{l}.540 \\
.690\end{array}$ & & \\
\hline $\begin{array}{l}\text { Roux-en-Y anastomosis MELD } \\
\text { score recipient }\end{array}$ & $\begin{array}{l}0.65(0.34-1.27) \\
1.01(0.95-1.08)\end{array}$ & $\begin{array}{l}.208 \\
.791\end{array}$ & & \\
\hline Donor type DCD & $3.02(1.82-4.76)$ & .000 & $2.67(1.47-4.87)$ & .001 \\
\hline $\begin{array}{l}\text { Preformed DSA Type I }(>5000) \\
\text { Preformed DSA Type II }(>5000)\end{array}$ & $\begin{array}{l}1.01(0.41-2.51) \\
0.62(0.15-2.56)\end{array}$ & $\begin{array}{l}.988 \\
.152\end{array}$ & & \\
\hline $\begin{array}{l}\text { Preformed DSA Type I (>1000) } \\
\text { Preformed DSA Type II (>1000) }\end{array}$ & $\begin{array}{l}1.22(0.52-2.82) \\
1.13(0.36-3.62)\end{array}$ & $\begin{array}{l}.650 \\
.832\end{array}$ & & \\
\hline $\begin{array}{l}\text { CIT } \\
\text { RWIT }\end{array}$ & $\begin{array}{l}1.00(0.99-1.01) \\
1.01(0.99-1.03)\end{array}$ & $\begin{array}{l}.507 \\
.221\end{array}$ & & \\
\hline
\end{tabular}

TABLE 3 Univariate and multivariate analyses of risk factors for NAS (all patients)

$\mathrm{HR}$, hazard ratio; $\mathrm{Cl}$, confidence intervals; OLT, orthotopic liver transplantation; $\mathrm{CMV}$, cytomegalovirus; DBD, donation after brain death; DCD, donation after circulatory death; BMI, body mass index; NAS, nonanastomotic biliary strictures; DSA, donor-specific antibodies against human leukocyte antigen (HLA) (mean fluorescence intensity (MFI)>5000). Significant values are printed bold.

${ }^{a}$ Viral, alcoholic, (PSC) primary sclerosing cholangitis, or other underlying liver disease. 
TAB LE 4 Univariate and multivariate analyses of risk factors for NAS (DCD excluded)

\begin{tabular}{|c|c|c|c|c|}
\hline & \multicolumn{2}{|l|}{ Univariate analysis } & \multicolumn{2}{|c|}{ Multivariate analysis } \\
\hline & HR $(95 \% \mathrm{Cl})$ & $P$-value & HR (95\% Cl) & $P$-value \\
\hline Donor age & $1.00(0.98-1.02)$ & .936 & & \\
\hline Donor gender male & $0.76(0.41-1.42)$ & .392 & & \\
\hline CMV IgG donor positive & $1.45(0.78-2.68)$ & .238 & & \\
\hline $\begin{array}{l}\text { CMV IgG recipient } \\
\text { positive }\end{array}$ & $1.30(0.62-2.74)$ & .491 & & \\
\hline Recipient age & $0.99(0.96-1.02)$ & .505 & & \\
\hline Recipient gender male & $1.06(0.58-1.94)$ & .858 & & \\
\hline Gender mismatch & $0.86(0.46-1.61)$ & .642 & & \\
\hline BMI donor & $1.01(0.92-1.12)$ & .802 & & \\
\hline OLT indication grouped ${ }^{\mathrm{a}}$ & $(0.28-2.08)$ & .897 & & \\
\hline OLT indication PSC & $1.02(0.50-2.08)$ & .964 & & \\
\hline Roux-en-Y anastomosis & $1.77(0.54-5.82)$ & .345 & & \\
\hline MELD score recipient & $1.02(0.91-1.13)$ & .784 & & \\
\hline $\begin{array}{l}\text { Preformed DSA Type I } \\
\text { (>5000) }\end{array}$ & $0.96(0.34-2.70)$ & .933 & & \\
\hline $\begin{array}{l}\text { Preformed DSA Type II } \\
(>5000)\end{array}$ & $0.52(0.12-2.15)$ & .366 & & \\
\hline $\begin{array}{l}\text { Preformed DSA Type I } \\
\text { (>1000) }\end{array}$ & $0.90(0.35-2.30)$ & .829 & & \\
\hline $\begin{array}{l}\text { Preformed DSA Type II } \\
(>1000)\end{array}$ & $1.31(0.32-5.46)$ & .708 & & \\
\hline CIT & $1.00(0.99-1.00)$ & .244 & & \\
\hline RWIT & $1.02(0.99-1.03)$ & .079 & $1.02(0.99-1.03)$ & .079 \\
\hline
\end{tabular}

$\mathrm{HR}$, hazard ratio; $\mathrm{Cl}$, confidence intervals; OLT, orthotopic liver transplantation; CMV, cytomegalovirus,DBD, donation after brain death; DCD, donation after circulatory death; BMI, body mass index; NAS, nonanastomotic biliary strictures; DSA, donor-specific antibodies against HLA (mean fluorescence intensity (MFI)>5000).

aViral, alcoholic, (PSC) primary sclerosing cholangitis, or other underlying liver disease. from the circulation at 12 months. Class II DSA were more likely to be persistent (data not shown). Importantly, in NAS patients, de novo DSA generally developed after the diagnosis of NAS (Figure 1, patients $1,2,5,7,8,9,10,11,12,13,14,16,19)$.

\section{3 | Donor-specific alloantibodies and graft survival}

In total, 19 (12.6\%) patients died and 15 (9.9\%) patients required a retransplantation during follow-up. Univariate and multivariate analyses of possible baseline risk factors for graft survival revealed male sex and donor BMI as independent risk factors for graft loss. Time-dependent Cox regression analysis identified NAS (aHR $=8.05$, $95 \% \mathrm{Cl} 3.28-19.77, P<.01)$ and de novo class II DSA (aHR $=2.84$, $95 \% \mathrm{Cl} 1.38-5.82, P<.01$ ) as independent risk factors for graft loss (Table 5). De novo DSA after OLT had a similar impact on graft survival in patients with NAS and in controls $(P=.10)$.

In the NAS group, 15 retransplantations were carried out for NAS with recurrent bacterial cholangitis $(n=13)$, infection unrelated to NAS $(n=1)$ and chronic rejection $(n=1)$. In the NAS group 13 patients died; causes of death were recurrent cholangitis $(n=1)$, chronic rejection ( $n=1)$, cholangitis from recurrent PSC $(n=1)$, pulmonary embolism ( $n=1)$, multi-organ failure $(n=1)$, malignancy $(n=2)$, infection unrelated to NAS ( $n=2)$, unknown ( $n=4)$. In the control group without NAS, no patient underwent retransplantation and six died; causes of death were infection ( $n=2)$, malignancy $(n=2)$, and unknown $(n=2)$.

\section{4 | DISCUSSION}

The present study demonstrated that both NAS and DSA against HLA class II-generated post-transplantation have a significant impact on graft survival. Yet, neither preformed, nor de novo DSA directed against class I or class II HLA antigens are associated with an increased risk for NAS development. Preformed DSA often disappear after OLT, while de novo DSA were more often generated after than before the diagnosis of NAS.

Antibody-mediated rejection (AMR) as a result of DSA is a serious event in kidney, lung, and heart transplant recipients, associated with an increased risk of graft loss. ${ }^{1,2,26}$ Conversely, liver allografts were presumed to be highly resistant to DSA, mainly due to the liver's ability to absorb circulating anti-HLA antibodies. Indeed, in the current study, preformed DSA usually disappeared within a year after OLT-with class II persisting longer than class I. This property was even presumed to be one of the factors providing a protective effect against 


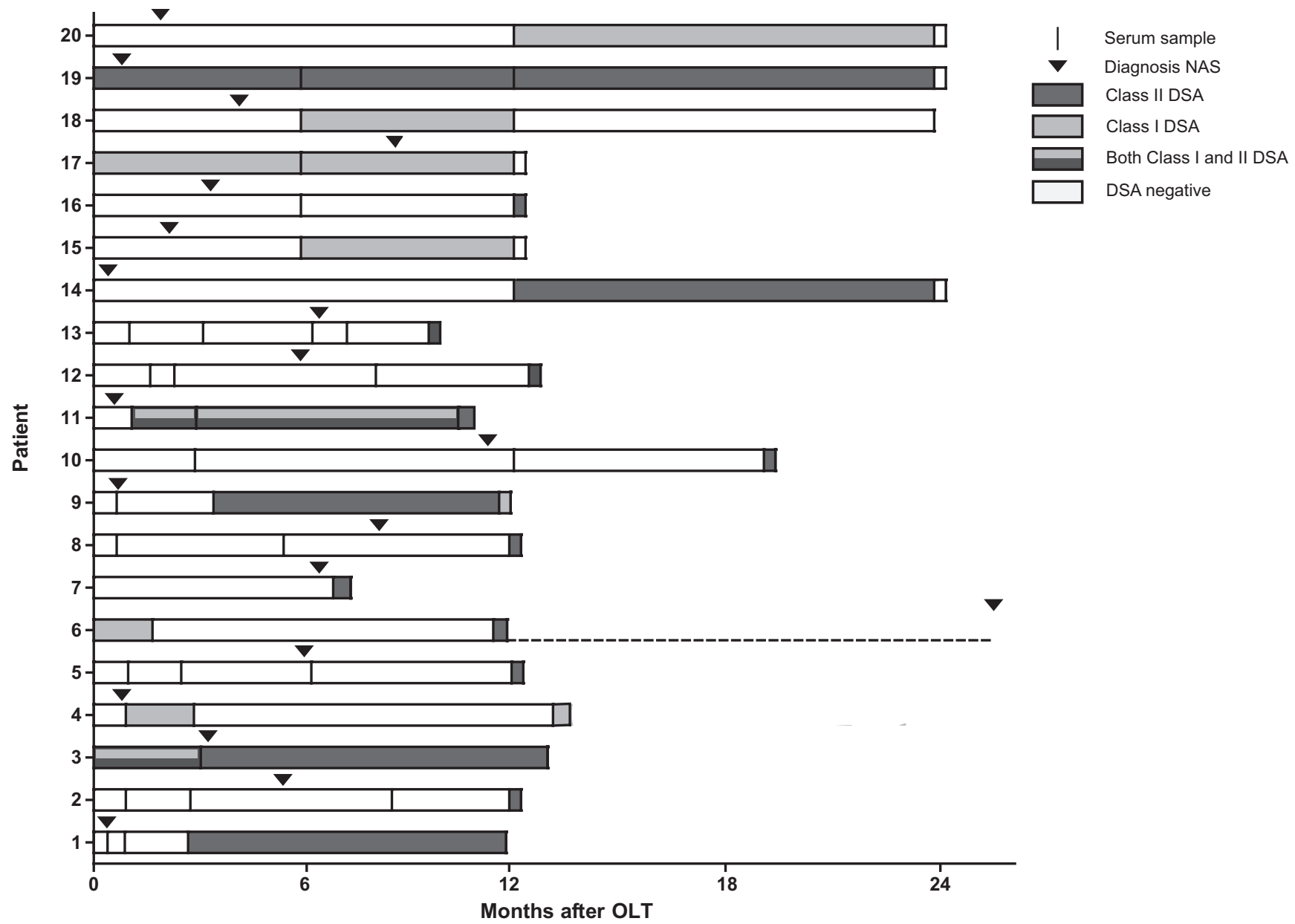

FIGURE 1 Donor-specific alloantibodies (DSA) status during follow-up in patients with nonanastomotic biliary strictures (NAS). Each patient is represented by a bar. The presence of class I and class II DSA is represented by different shades of gray. Repeated measurements were only available in a subgroup of NAS patients $(n=20)$

acute renal allograft rejection in simultaneous liver-kidney transplantations and may be the result of release of soluble MHC complexes from the liver. ${ }^{27}$ However, a recent study demonstrated that renal allograft protection by the liver is incomplete in case of preformed class II DSA and that, subsequently, despite some protection both grafts remain at risk for rejection. ${ }^{28}$ In addition, preformed and de novo DSA are increasingly recognized as a cause of rejection and allograft loss after OLT. ${ }^{7,8}$ This is probably the result of the ability of DSA to bind to endothelial cells in the portal triads leading to complement activation. Consistent with previous literature, ${ }^{8,29}$ DSA formed after OLT were, in the majority of cases, directed against class II antigens, mainly against HLA-DQ. Serial measurements demonstrated that antibodies against class II antigens tended to appear later after transplantation and to remain longer in the circulation compared to antibodies against class I antigens. Class II DSA have previously been associated with graft loss. ${ }^{30}$ The present study shows that after OLT, this is only the case for de novo class II DSA. The prevalence of preformed and de novo DSA in this study cohort was $9.9 \%$ and $21.2 \%$, respectively. This is consistent with previous studies in which preformed DSA were detected in 5\%$22 \%$ of the liver transplantation candidates. ${ }^{7,31,32}$ For de novo DSA, the reported prevalence varies between $8 \%$ and $24 \% .8,29,33$
Bile duct complications after OLT are frequent and a major cause of morbidity and mortality. The role of DSA in the occurrence of bile duct complications after transplantation was unclear. Under physiological conditions, biliary epithelial cells highly express HLA class I antigens, whereas there is only a weak expression of class II antigens. ${ }^{34}$ However, as a result of ischemia during OLT, a sterile immune response is initiated, with elevated levels of proinflammatory cytokines (eg, interferon- $\gamma$ and interleukins) and infiltration of inflammatory cells into the biliary epithelium, which can result in an upregulation of HLA class II antigens. ${ }^{21,35,36}$ Ischemiareperfusion injury (IRI) of the biliary epithelium is also considered as one of the most important risk factors for NAS development. The effect of IRI is not limited to cholangiocytes only. In a previous study, we showed that severe IRI to hepatocytes, as reflected by a high peak alanine aminotransferase, was also an important risk factor for NAS development. ${ }^{10}$ Interestingly, Gugenheim et al demonstrated an upregulation of hepatic class I antigen expression after a period of normothermic ischemia in a rat model. ${ }^{37}$

It could therefore be expected that a relation exists between IRI, the presence of antibodies against donor HLA antigens expressed on hepatocytes and biliary epithelium and bile duct complications, 
TABLE 5 Univariate and multivariate analyses of pre-OLT risk factors for graft loss

\begin{tabular}{|c|c|c|c|c|c|}
\hline \multirow[b]{2}{*}{ Variables } & & \multicolumn{2}{|l|}{ Univariate analysis } & \multicolumn{2}{|c|}{ Multivariate analysis } \\
\hline & & $\operatorname{HR}(95 \% \mathrm{CI})$ & $P$-value & $\mathrm{HR}(95 \% \mathrm{Cl})$ & $P$-value \\
\hline $\begin{array}{l}\text { Recipient age } \\
\text { at OLT }\end{array}$ & Continuous & $1.02(0.99-1.05)$ & .254 & & \\
\hline $\begin{array}{l}\text { Recipient } \\
\text { gender }\end{array}$ & Male & $2.47(1.01-6.06)$ & .049 & $2.69(1.07-6.79)$ & .036 \\
\hline Recipient BMI & Continuous & $1.04(0.96-1.13)$ & .320 & & \\
\hline Donation & $\mathrm{DCD}$ & $1.35(0.55-3.35)$ & .515 & & \\
\hline Donor age & Continuous & $1.02(0.99-1.04)$ & .259 & & \\
\hline Donor gender & Male & $1.35(0.65-2.78)$ & .420 & & \\
\hline Donor BMI & Continuous & $1.11(0.99-1.25)$ & .063 & $1.09(0.96-1.24)$ & .175 \\
\hline CMV mismatch & Yes (D+/R-) & $0.65(0.29-1.45)$ & .288 & & \\
\hline Rejection & Yes & $0.80(0.30-2.10)$ & .650 & & \\
\hline Pre-OLT DSA & Class I & $1.69(0.22-12.92)$ & .612 & & \\
\hline Pre-OLT DSA & Class II & $0.73(0.10-5.51)$ & .760 & & \\
\hline NAS & $\begin{array}{l}\text { Time- } \\
\text { dependent }\end{array}$ & & & $8.05(3.28-19.77)$ & $<.010$ \\
\hline $\begin{array}{l}\text { De novo DSA } \\
\text { class II }\end{array}$ & $\begin{array}{l}\text { Time- } \\
\text { dependent }\end{array}$ & & & $2.84(1.38-5.82)$ & $<.010$ \\
\hline
\end{tabular}

$\mathrm{HR}$, hazard ratio; $\mathrm{Cl}$, confidence intervals; OLT, orthotopic liver transplantation; $\mathrm{CMV}$, cytomegalovirus, DBD, donation after brain death; DCD, donation after circulatory death; BMI, body mass index; NAS, nonanastomotic biliary strictures. Significant values are printed bold. such as the development of nonanastomotic biliary strictures. In the present study, we could not find an association between preformed or de novo generated DSA and NAS. According to the present data, de novo DSA were also not related to NAS. De novo DSA-most of class II-generally developed after the diagnosis of NAS, supporting the conclusion that de novo DSA may worsen NAS, but probably are not involved in the initiation of NAS. This is in accordance with a study performed by lacob et al. ${ }^{38}$ In that study, post-transplant DSA in relation to both anastomotic and nonanastomotic biliary strictures was evaluated. The presence of post-transplant HLA class II DSA was related to the development of anastomotic strictures, but no association was found between the presence of alloantibodies and the occurrence of NAS. Yet, because of the cross-sectional design of that study, it remained unclear whether these DSA were persistent or formed after transplantation, and several studies-including the current one-have demonstrated that predominantly de novo DSA are relevant in predicting transplantation outcome. ${ }^{8,9}$ Furthermore, the levels of DSA were not described by lacob et al. In the present study, only DSA with an MFI of $>5000$ were considered positive, as mainly high MFI DSA are related to an inferior clinical outcome after OLT. ${ }^{7,9}$ Sensitivity analysis was performed in the current study for MFI>1000 with similar outcomes. Other studies regarding this topic were not specific to NAS. For example, Takeya et al found an association between DSA and heterogeneous group of bile ducts complications, consisting of patients with biliary obstructions, biliary strictures and focal necrosis of the bile ducts. ${ }^{23}$ DSA have also been suggested to be related to ductopenia, ${ }^{39}$ which is most likely the result of chronic and irreversible rejection. ${ }^{22,40,41}$
A limitation of the present study is that from the majority of patients post-transplant DSA could only be measured 1 year after transplantation. Therefore, the influence of de novo DSA that are transiently present, but are cleared from the circulation in the first year after transplantation, may have been missed in our analysis. This might have resulted in underestimation of the contribution of transient de novo class I DSA, which in a subgroup of patients that we analyzed longitudinally developed early after OLT but were cleared from the circulation within the first year after transplantation. Therefore, a prospective study with repeated DSA measurements during follow-up is required to provide information on a possible association between transiently appearing de novo DSA and NAS development.

Another problem is that radiodiagnostic features of NAS resemble the diagnostic criteria for PSC. Therefore, it may be difficult to distinguish NAS and recurrence of PSC after OLT. As graft failure occurred in only four PSC patients (two without DSA and two with de novo class II DSA), and as PSC was not a risk factor for NAS in this study, we consider it unlikely that this has influenced our results.

According to the original Banff guidelines, the diagnosis of antibody-mediated rejection after kidney transplantation requires three criteria: morphologic evidence of tissue injury, serologic evidence of DSA formation or other anti-donor endothelial antigens, and evidence of antibody interaction with vascular endothelium, for example, C4d deposition in the peritubular capillaries. ${ }^{42}$ Recently, the criteria were revised, and acute and chronic antibody-mediated rejection may now be diagnosed in the absence of $\mathrm{C} 4 \mathrm{~d}$ deposition. However, in this case, additional evidence of current or recent antibody interaction with the vascular endothelium must be present. ${ }^{43}$ 
In conclusion, the present study is the first study that assessed the relationship of DSA with nonanastomotic biliary stricture development and graft survival after orthotopic liver transplantation. Neither preformed DSA nor de novo DSA generated within the first year after liver transplantation were associated with the development of NAS. However, the time-dependent analysis revealed that both NAS and de novo class II DSA developing after liver transplantation were independently associated with graft loss.

\section{ACKNOWLEDGEMENTS}

The authors would like to thank A. van der Eijk for providing serum samples.

\section{CONFLICT OF INTEREST}

The authors declare no conflict of interest.

\section{AUTHORS' CONTRIBUTIONS}

Anne Claire den Dulk, Xiaolei Shi, Bart van Hoek, Jaap Kwekkeboom and Dave L. Roelen: Participated in research design, performance of the research, data analysis, writing of the paper; Cornelia. J. Verhoeven, Jeroen Dubbeld, Simone H. Brand-Schaaf, Arantza Fariña Sarasqueta, Luc J.W. van der Laan and Herold J. Metselaar: Participated in performance of the research; Frans H.J. Claas: Participated in research design, performance of the research, writing of the paper; Ron Wolterbeek: Participated in data analysis; Hein W. Verspaget: Participated in performance of the research, design of the study.

\section{ORCID}

Bart van Hoek iD http://orcid.org/0000-0001-6527-764X

\section{REFERENCES}

1. Witt CA, Gaut JP, Yusen RD, et al. Acute antibody-mediated rejection after lung transplantation. J Heart Lung Transplant. 2013;32:1034-1040.

2. Devos JM, Gaber AO, Teeter LD, et al. Intermediate-term graft loss after renal transplantation is associated with both donor-specific antibody and acute rejection. Transplantation. 2014;97:534-540.

3. Gugenheim J, Le TB, Rouger P, et al. Relationship between the liver and lymphocytotoxic alloantibodies in inbred rats. Specific absorption by nonparenchymal liver cells. Transplantation. 1988;45:474-478.

4. Astarcioglu I, Gugenheim J, Crafa F, Saint Paul MC, Reynes M. Hyperacute rejection of liver allografts in sensitized rats: role of nonparenchymal liver cells. J Surg Res. 1995;58:182-188.

5. Della-Guardia B, Almeida MD, Meira-Filho SP, et al. Antibodymediated rejection: hyperacute rejection reality in liver transplantation? A case report. Transplant Proc. 2008;40:870-871.

6. Hale DA. Basic transplantation immunology. Surg Clin North Am. 2006;86:1103-1125.

7. O'Leary JG, Kaneku H, Jennings LW, et al. Preformed class II donorspecific antibodies are associated with an increased risk of early rejection after liver transplantation. Liver Transpl. 2013;19:973-980.
8. Kaneku H, O'Leary JG, Banuelos N, et al. De novo donor-specific HLA antibodies decrease patient and graft survival in liver transplant recipients. Am J Transplant. 2013;13:1541-1548.

9. O'Leary JG, Kaneku H, Susskind BM, et al. High mean fluorescence intensity donor-specific anti-HLA antibodies associated with chronic rejection Postliver transplant. Am J Transplant. 2011;11:1868-1876.

10. den Dulk AC, Sebib Korkmaz K, de Rooij BJ, et al. High peak alanine aminotransferase determines extra risk for nonanastomotic biliary strictures after liver transplantation with donation after circulatory death. Transpl Int. 2015;28:492-501.

11. Ten Hove WR, Sebib Korkmaz K, op den Dries S, et al. Matrix metalloproteinase 2 genotype is associated with nonanastomotic biliary strictures after orthotopic liver transplantation. Liver Int. 2011;31:1110-1117.

12. Buis $\mathrm{Cl}$, Hoekstra H, Verdonk RC, Porte RJ. Causes and consequences of ischemic-type biliary lesions after liver transplantation. J Hepatobiliary Pancreat Surg. 2006;13:517-524.

13. Karimian N, Westerkamp AC, Porte RJ. Biliary complications after orthotopic liver transplantation. Curr Opin Organ Transplant. 2014;19:209-216.

14. Chan EY, Olson LC, Kisthard JA, et al. Ischemic cholangiopathy following liver transplantation from donation after cardiac death donors. Liver Transpl. 2008;14:604-610.

15. Buis $\mathrm{Cl}$, Geuken $\mathrm{E}$, Visser DS, et al. Altered bile composition after liver transplantation is associated with the development of nonanastomotic biliary strictures. J Hepatol. 2009;50:69-79.

16. op den Dries S, Sutton ME, Lisman T, Porte RJ. Protection of bile ducts in liver transplantation: looking beyond ischemia. Transplantation. 2011;92:373-379.

17. op den Dries $\mathrm{S}$, Buis $\mathrm{Cl}$, Adelmeijer J, et al. The combination of primary sclerosing cholangitis and CCR5-Delta32 in recipients is strongly associated with the development of nonanastomotic biliary strictures after liver transplantation. Liver Int. 2011;31:1102-1109.

18. Rull R, Garcia Valdecasas JC, Grande L, et al. Intrahepatic biliary lesions after orthotopic liver transplantation. Transpl Int. 2001;14:129-134.

19. Halme L, Hockerstedt K, Lautenschlager I. Cytomegalovirus infection and development of biliary complications after liver transplantation. Transplantation. 2003;75:1853-1858.

20. Hoekstra $\mathrm{H}$, Buis $\mathrm{Cl}$, Verdonk RC, et al. Is Roux-en-Y choledochojejunostomy an independent risk factor for nonanastomotic biliary strictures after liver transplantation? Liver Transpl. 2009;15:924-930.

21. Ayres RC, Neuberger JM, Shaw J, Joplin R, Adams DH. Intercellular adhesion molecule- 1 and $\mathrm{MHC}$ antigens on human intrahepatic bile duct cells: effect of pro-inflammatory cytokines. Gut. 1993;34:1245-1249.

22. Batts KP, Moore SB, Perkins JD, Wiesner RH, Grambsch PM, Krom RA. Influence of positive lymphocyte crossmatch and HLA mismatching on vanishing bile duct syndrome in human liver allografts. Transplantation. 1988;45:376-379.

23. Takaya S, Jain A, Yagihashi A, et al. Increased bile duct complications and/or chronic rejection in crossmatch positive human liver allografts. Transplant Proc. 1999;31:2028-2031.

24. Demetris AJ, Nakamura K, Yagihashi A, et al. A clinicopathological study of human liver allograft recipients harboring preformed IgG lymphocytotoxic antibodies. Hepatology. 1992;16:671-681.

25. Sakashita H, Haga H, Ashihara E, et al. Significance of C4d staining in ABO-identical/compatible liver transplantation. Mod Pathol. 2007;20:676-684.

26. Frank R, Molina MR, Wald JW, Goldberg LR, Kamoun M, Lal P. Correlation of circulating donor-specific anti-HLA antibodies and presence of $\mathrm{C} 4 \mathrm{~d}$ in endomyocardial biopsy with heart allograft outcomes: a single-center, retrospective study. J Heart Lung Transplant. 2013;32:410-417.

27. Simpson N, Cho YW, Cicciarelli JC, Selby RR, Fong TL. Comparison of renal allograft outcomes in combined liver-kidney transplantation versus subsequent kidney transplantation in liver 
transplant recipients: analysis of UNOS database. Transplantation. 2006;82:1298-1303.

28. O'Leary JG, Gebel HM, Ruiz R, et al. Class II alloantibody and mortality in simultaneous liver-kidney transplantation. Am J Transplant. 2013;13:954-960.

29. Del Bello A, Congy-Jolivet N, Danjoux M, et al. De novo donorspecific anti-HLA antibodies mediated rejection in liver-transplant patients. Transpl Int. 2015;28:1371-1382.

30. lacob S, Cicinnati VR, Lindemann M, et al. Donor-specific anti-HLA antibodies and endothelial C4d deposition-association with chronic liver allograft failure. Transplantation. 2015;99:1869-1875.

31. Taner T, Gandhi MJ, Sanderson SO, et al. Prevalence, course and impact of HLA donor-specific antibodies in liver transplantation in the first year. Am J Transplant. 2012;12:1504-1510.

32. Muro M, Marin L, Miras M, et al. Liver recipients harbouring antidonor preformed lymphocytotoxic antibodies exhibit a poor allograft survival at the first year after transplantation: experience of one centre. Transpl Immunol. 2005;14:91-97.

33. Fontana M, Moradpour D, Aubert V, Pantaleo G, Pascual M. Prevalence of anti-HLA antibodies after liver transplantation. Transpl Int. 2010;23:858-859.

34. Barbatis C, Woods J, Morton JA, Fleming KA, McMichael A, McGee JO. Immunohistochemical analysis of $\operatorname{HLA}(A, B, C)$ antigens in liver disease using a monoclonal antibody. Gut. 1981;22: 985-991.

35. Demetris AJ, Lasky S, Van Thiel DH, Starzl TE, Whiteside T. Induction of DR/IA antigens in human liver allografts. An immunocytochemical and clinicopathologic analysis of twenty failed grafts. Transplantation. 1985;40:504-509.

36. Fontes $\mathrm{P}$, Lopez $\mathrm{R}$, van der Plaats $\mathrm{A}$, et al. Liver preservation with machine perfusion and a newly developed cell-free oxygen carrier solution under subnormothermic conditions. Am J Transplant. 2015;15:381-394.
37. Gugenheim J, Reynes M, Crafa F, Saint-Paul MC, Fabiani B, Mouiel J. Normothermic ischemia induces major histocompatibility complex class I expression in hepatocytes. Eur Surg Res. 1996;28:256-261.

38. lacob S, Cicinnati VR, Dechene A, et al. Genetic, immunological and clinical risk factors for biliary strictures following liver transplantation. Liver Int. 2012;32:1253-1261.

39. Musat Al, Agni RM, Wai PY, et al. The significance of donor-specific HLA antibodies in rejection and ductopenia development in ABO compatible liver transplantation. Am J Transplant. 2011;11:500-510.

40. Wiesner RH, Ludwig J, van Hoek B, Krom RA. Current concepts in cell-mediated hepatic allograft rejection leading to ductopenia and liver failure. Hepatology. 1991;14:721-729.

41. van Hoek B, Wiesner RH, Krom RA, Ludwig J, Moore SB. Severe ductopenic rejection following liver transplantation: incidence, time of onset, risk factors, treatment, and outcome. Semin Liver Dis. 1992;12:41-50.

42. Racusen LC, Colvin RB, Solez K, et al. Antibody-mediated rejection criteria - an addition to the Banff 97 classification of renal allograft rejection. Am J Transplant. 2003;3:708-714.

43. Haas M, Sis B, Racusen LC, et al. Banff 2013 meeting report: inclusion of c4d-negative antibody-mediated rejection and antibodyassociated arterial lesions. Am J Transplant. 2014;14:272-283.

How to cite this article: den Dulk AC, Shi X, Verhoeven CJ, et al. Donor-specific anti-HLA antibodies are not associated with nonanastomotic biliary strictures but both are independent risk factors for graft loss after liver transplantation. Clin Transplant. 2017;e13163. https://doi. org/10.1111/ctr.13163 